\title{
Possibilités d'action publique pour une meilleure prise en compte des marchés domestiques du bois dans le cadre des accords de partenariat volontaire (APV) au Cameroun
}

\author{
Paolo Omar Cerutti, Guillaume Lescuyer, Raphaël Tsanga, Samuel Assembe Mvondo, \\ Edouard Essiane et Andrew Wardell
}

\section{Points essentiels}

- L'Accord sur l'application des réglementations forestières, la gouvernance et les échanges commerciaux (FLEGT) a été lancé en 2003 par l'Union européenne (UE) afin de lutter au niveau mondial contre l'exploitation forestière illégale. En particulier, le FLEGT vise à réduire le commerce du bois récolté illégalement entre l'UE et les pays partenaires producteurs de bois.

- Le FLEGT fonctionne grâce à deux principaux instruments : des accords commerciaux bilatéraux — appelés accords de partenariat volontaire (APV) - qui sont signés avec les pays producteurs qui le souhaitent et le règlement sur le bois de I'Union européenne qui est entré en vigueur en mars 2013. Ce règlement sur le bois de l'UE impose aux importateurs européens de faire preuve d'une diligence raisonnable lorsqu'ils s'approvisionnent en bois à l'étranger pour se prémunir contre les produits illégaux.

- À ce jour, six pays ont signé un APV. Cinq d'entre eux se sont même engagés à appliquer les dispositions de cet APV concernant la vérification de la légalité non seulement au bois exporté vers l'Europe, mais aussi à celui qui est commercialisé sur leurs marchés intérieurs. Ceci signifie que le bois récolté et commercialisé sur le marché domestique sera réglementé par des programmes de permis nationaux dans le cadre de l'APV (c'est le système de garantie de la légalité du bois ou Timber Legality Assurance System, TLAS).

- Les résultats de Pro-Formal indiquent que le Cameroun se caractérise par un secteur domestique du bois important, dynamique et en grande partie informel, qui contribue à faire vivre localement des milliers d'utilisateurs de la forêt, notamment de petits paysans, des populations autochtones, des scieurs artisanaux, des négociants et des prestataires de service.

- Le secteur domestique du bois se caractérise par les activités de petits exploitants agricoles, de scieurs artisanaux et de négociants qui possèdent rarement un permis de coupe légal, abattent et scient de petites quantités de bois à l'aide d'une tronçonneuse ou d'une scie mobile. Ce bois, qui n'est pas d'une grande qualité, est vendu sur les marchés domestiques ou de l'autre côté des frontières dans les pays voisins (p. ex. Tchad et Nigéria). Il fait rarement l'objet d'une taxation formelle.

- En revanche, la taxation informelle est omniprésente le long de la chaîne de production. Selon les résultats, les opérateurs artisanaux versent environ $9 \%$ de leurs marges, soit près de 6 millions d'EUR par an en pots-de-vin aux représentants des ministères, à la police locale, aux militaires et aux douaniers.

- En signant l'APV, le Cameroun s'est engagé à entreprendre de grandes réformes de gouvernance dans tout le secteur forestier. Les lois existantes ne favorisent pas la vitalité du marché domestique du bois à petite échelle. D'après les conclusions de Pro-Formal, il est nécessaire de faciliter et de simplifier l'accès à la ressource, de mettre au point et d'adopter des régimes fiscaux spécifiques au secteur domestique du bois (tels que des redevances, des taxes sur la transformation, le transport et la commercialisation), de faire progresser l'accès au crédit à des conditions favorables pour les petits opérateurs, d'améliorer la diffusion des informations auprès de ceux-ci et de créer des incitations en vue du respect de la loi.

- Au Cameroun, comme dans les autres pays APV, les usagers des forêts et les négociants au niveau local ont été généralement absents des négociations nationales sur l'Accord. Cela est dû à leur grand nombre, à leur éparpillement sur un vaste territoire et à une capacité d'organisation médiocre, ainsi qu'aux tensions qui existent souvent entre la volonté affichée des pouvoirs publics de les faire sortir du secteur informel et le comportement de fonctionnaires décentralisés à la recherche de rentes. Par conséquent, avant d'amorcer des réformes, des discussions officielles et périodiques doivent être organisées pour échanger avec les scieurs artisanaux et les négociants sur leurs besoins propres, les modes de gouvernance coutumière et les difficultés et attentes éventuelles par rapport à la formalisation.

- De même, l'implication des autres ministères, en particulier ceux des Finances, de l'Agriculture et de l'Administration territoriale doit se poursuivre, car elle est nécessaire pour un débat national inclusif à propos des répercussions du fonctionnement de l'APV (p. ex. financières et foncières) sur les opérateurs, surtout les petits. Cependant, en raison de retards politiques et techniques, ce débat n'a pas été systématique. Cela pourrait entraîner l'échec de la mise en œuvre si l'on ne pèse pas dès le départ les avantages et les inconvénients du système (p. ex. les coûts pour les opérateurs). 


\section{Contexte}

Le secteur forestier au Cameroun est régi par la loi de 1994 portant régime des forêts et son décret d'application de 1995,' suivis de dizaines de règlements, d'arrêtés et de procédures officielles. La loi de 1994 visait à favoriser un aménagement durable des forêts tout en augmentant la contribution du secteur forestier à l'économie nationale. Ce secteur représente environ $4 \%$ du PIB (CIFOR et MINFOF 2013), fait rentrer près de 62 millions EUR de recettes annuelles dans les coffres de l'État et entretient environ 13000 emplois formels et 45000 emplois informels directs.

La loi de 1994 faisait suite à une politique forestière de 1993 dont l'objectif était d'encourager les populations locales à travailler dans le secteur forestier et de veiller à ce qu'elles perçoivent une partie des recettes générées. La loi a ainsi introduit les forêts communautaires - qui pouvaient être créées et exploitées par les communautés rurales en suivant un plan simplifié de gestion et un mécanisme de partage des bénéfices - qui prévoyait que $50 \%$ de la nouvelle redevance forestière annuelle payée par les sociétés d'exploitation forestière seraient redistribués aux conseils et aux villages voisins des unités forestières d'aménagement. En dépit des objectifs affichés, la loi et ses décrets d'application restent très focalisés sur la réglementation du secteur forestier industriel à grande échelle orienté vers l'exportation.

La loi énumère plusieurs permis pour les petits opérateurs : (1) Le permis de bois d'œuvre (PEBO), qui est un permis de coupe valable pour un an et un volume de $500 \mathrm{~m}^{3}$; (2) L'autorisation personnelle de coupe valable pour trois mois et un volume de $30 \mathrm{~m}^{3}$; (3) Un droit d'usage pour les populations vivant dans les villages voisins des zones forestières. Les options 2 et 3 ne doivent pas servir à des fins commerciales, et l'option 1 a été suspendue pendant plusieurs années et jamais adaptée aux besoins des usagers auxquels elle était destinée, c.-à-d. les petits opérateurs qui, de leur côté, en font rarement la demande (Cerutti et Tacconi 2008 ; Cerutti et Lescuyer 2011).

Les résultats de la recherche Pro-Formal indiquent que la grande majorité des petits opérateurs exploite le bois et approvisionne le marché domestique sans document d'exploitation valide (Cerutti et Lescuyer 2011). Les résultats permettent aussi de constater que le personnel du ministère des Forêts et de la Faune (MINFOF), qui est responsable de la réglementation du secteur, a mis au point un vaste système informel pour soutirer de l'argent aux scieurs artisanaux (Cerutti et al. 2013).

Quand il voudra mettre en œuvre l'APV, le Cameroun sera confronté à un certain nombre de difficultés : la demande de bois en augmentation aux niveaux régional et national, la contribution positive du secteur à l'économie locale qui devra être soutenue, l'absence de réglementation adéquate en termes d'accès à la ressource, d'exploitation et de vente, et enfin, les intérêts en jeu qui se sont développés au fil du temps.

Signé en 2010 avec I'UE, I'APV promet un cadre juridique qui couvre l'ensemble de la production nationale de bois, dont celle destinée au marché domestique. Cependant, l'annexe II de I'APV,

1 Loi N 94/01 du 20 janvier 1994 portant régime des forêts, de la faune et de la pêche ; Décret Nº5/531/PM du 23 août 1995 qui donne la liste des titres et autorisations d'exploitation pour lesquels des grilles de légalité ont été préparées, n'évoque pas le PEBO, seule autorisation légale prévue pour les scieurs artisanaux.

Cette annexe II énonce que « d'autres grilles de légalité seront construites au cours de la phase d'opérationnalisation du système dès lors que leur pertinence sera établie » (République du Cameroun et Union européenne 2010). Les conclusions quantitatives et qualitatives du projet Pro-Formal révèlent que la pertinence du secteur est déjà avérée et qu'une actualisation de l'annexe II devrait être envisagée immédiatement (Tableau 1). Cela permettrait de maintenir le marché domestique du bois dans le cadre du programme politique et technique de l'APV. L'existence de grilles de légalité pour tous les titres permettrait aux futurs auditeurs du système de garantie de la légalité du bois de vérifier les activités réalisées en vue d'approvisionner le marché domestique du bois.

\section{Tableau 1. Principales conclusions quantitatives - Cameroun}

\begin{tabular}{lr}
\hline & Sciages (par an, $\mathrm{m}^{3}$ ) \\
\hline $\begin{array}{l}\text { Consommation - marché } \\
\text { domestique }(2008-2012)^{\mathrm{a}}\end{array}$ & $850000-950000$ \\
\hline
\end{tabular}

Exportations régionales par 80000 de petits négociants vers le Tchad et le Nigéria ${ }^{a}$

Déchets de scierie industrielle

(vendus sur le marché domestique) ${ }^{\mathrm{a}}$

Production informelle des scieurs artisanaux (20082012)

Production moyenne des forêts communautaires ${ }^{\mathrm{b}}$

Exportations (industrielle, officielle, sciages, 2008-2012)

Personnes employées par le secteur informel du bois ${ }^{a}$

Contribution à l'économie

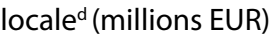

Bénéfice des scieurs artisanaux par $\mathrm{m}^{3}$ exploité (EUR)

Marge bénéficiaire

a D'après les enquêtes Pro-Formal (marge bénéficiaire exprimée en pourcentage du prix de vente).

b Fondé sur l'hypothèse d'un nombre annuel de Certificats Annuels d'Exploitation (CAE) délivrés aux forêts communautaires exploitées au nombre de 150, produisant chacune en moyenne $200 \mathrm{~m} 3$ par an.

c Données du ministère des Forêts et de la Faune.

d Valeur agrégée des salaires, redevances et des bénéfices.

Les facteurs politiques et techniques qui font que la majorité de la production reste informelle ont été discutés lors d'une série 


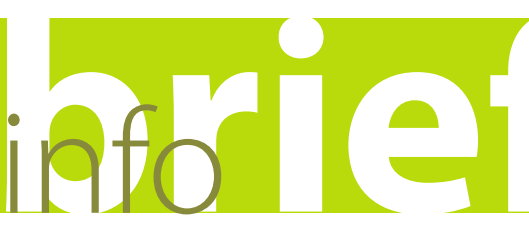

No. 90

Septembre 2014

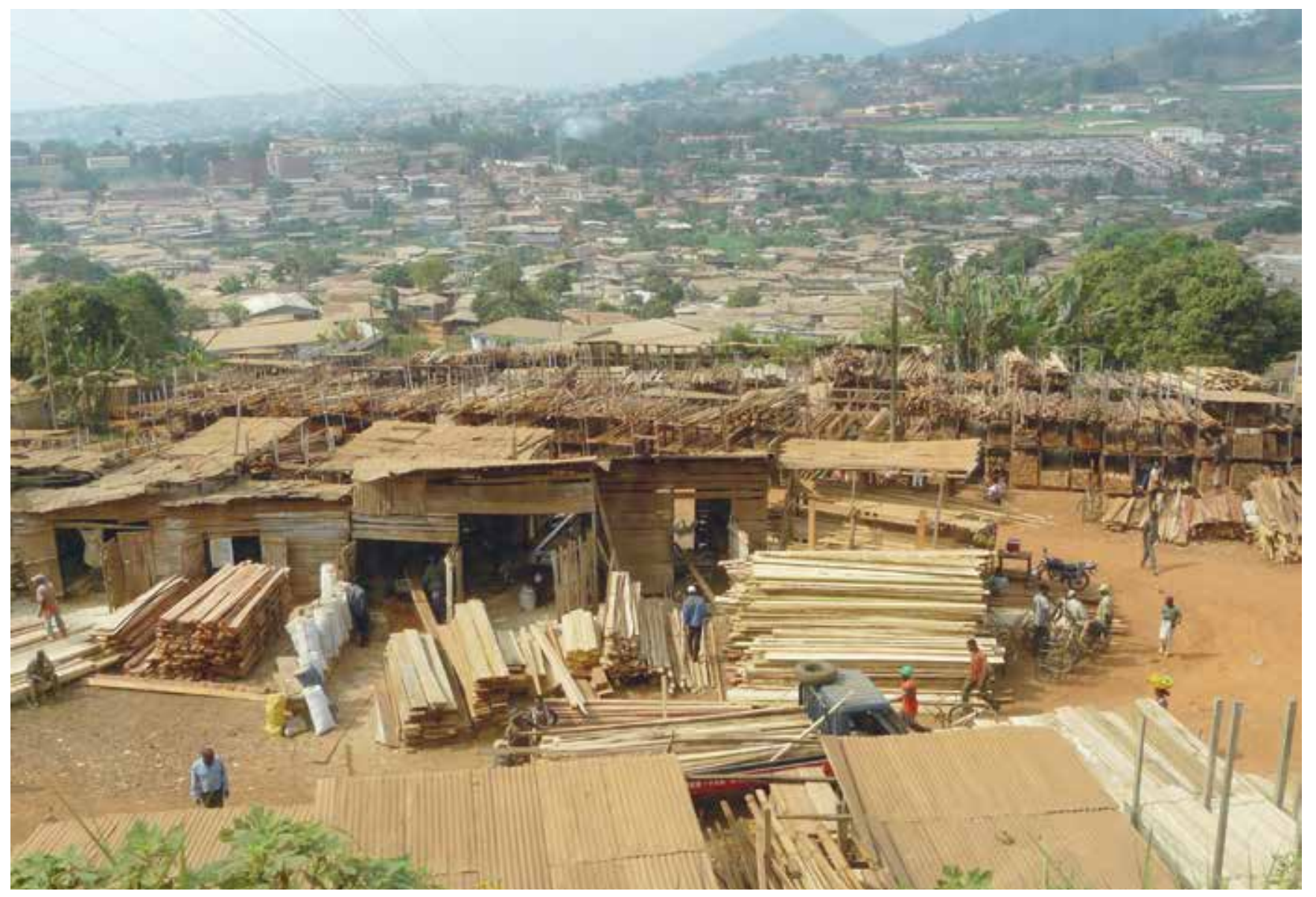

\section{Marché du bois au Cameroun}

Photo Charlie Pye-Smith, CIFOR

d'ateliers nationaux avec les parties concernées (gouvernement camerounais, société civile, opérateurs formels et informels et la communauté des bailleurs de fonds). Ces réunions ont débouché sur un éventail de possibilités d'action énumérées ci-dessous.

\section{Possibilités d'action}

Problème La majorité des scieurs artisanaux ne demande pas la seule autorisation qui existe actuellement (c.-à-d. le PEBO).

\section{Recommandations}

- La Direction des Forêts au MINFOF devrait commencer à enregistrer les opérateurs actuels dans chaque arrondissement pour leur accorder un agrément professionnel simplifié (et éventuellement gratuit) et une autorisation légale d'exploitation (p. ex. des PEBO faciles à obtenir). Le principal objectif de ce processus serait de délivrer au moins quelques titres immédiatement aux opérateurs enregistrés ( $p$. ex. dans une zone pilote à déterminer parmi les principaux fournisseurs de bois), tout en intégrant davantage d'opérateurs au fil du temps et au fur et à mesure que les connaissances augmentent sur la demande et les ressources disponibles.

- Prolonger la validité des autorisations (p. ex. pendant trois ans) ou maintenir la quantité de bois ou la zone d'exploitation comme les deux seules limitations pour les autorisations destinées aux petits opérateurs. De cette manière, ceux-ci pourraient récolter les quantités autorisées sur un délai à leur convenance. Le respect des volumes autorisés sera contrôlé par la vérification en bonne et due forme des lettres de voiture délivrées par le MINFOF et portées par chaque titulaire d'autorisation.

- Un « délai de grâce » devrait être convenu pendant lequel les ventes aux enchères et l'exploitation seront testées sans sanction. Celui-ci devra être suffisamment long pour que le MINFOF ait confiance dans l'efficacité du projet, mais pas trop afin de ne pas donner d'habitudes. Étant donné la dynamique actuelle du secteur, nous pensons qu'un délai de six mois à un an devrait suffire pour tester et mettre en œuvre le système dans une zone pilote.

Problème Le mode d'accès aux arbres actuel est illégal.

\section{Recommandation}

- La «propriété » individuelle et coutumière des arbres devrait être reconnue dans le domaine forestier non permanent (DFNP), à l'exclusion des cas où d'autres titres d'exploitation ou permis sont accordés, comme les forêts communautaires, ventes de volume sur pied, autorisation de récupération de bois. Cela a pour but de garantir que, en l'absence de titre d'exploitation ou de permis formellement octroyé par l'État, les arbres du DFNP appartiennent à un « propriétaire » coutumier officiellement reconnu qui peut les vendre. 


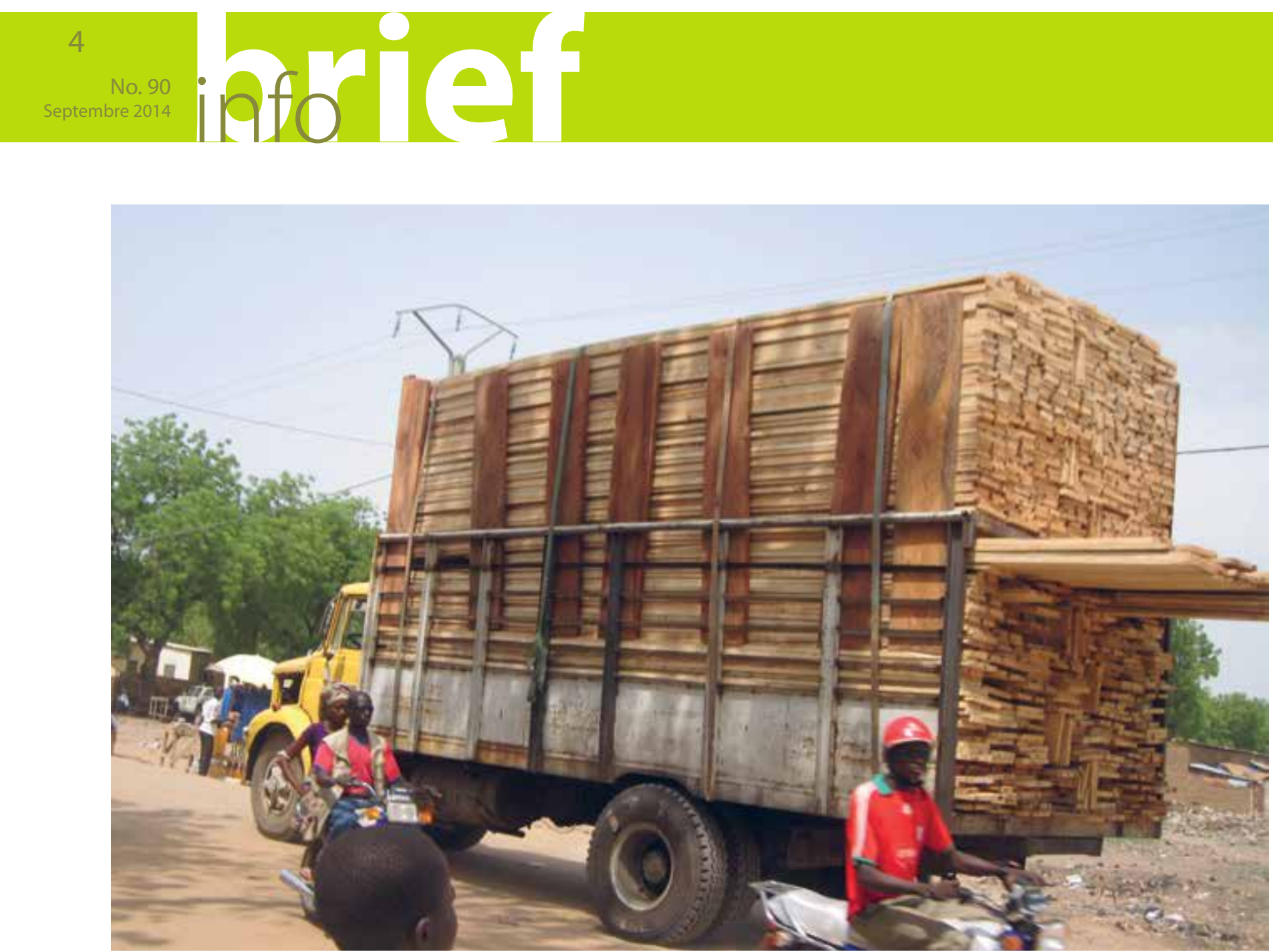

\section{Bois en direction de la frontière nord avec le Tchad}

Photo Emmanuel Damboya, CIFOR

Problème Le cadre juridique actuel n'autorise pas le droit d'usage à but lucratif. En pratique, cependant, les arbres sont vendus au quotidien par des «propriétaires » coutumiers à des négociants en bois ou des scieurs artisanaux, pour être revendus ensuite sur le marché domestique.

\section{Recommandation}

- Le cadre juridique devrait être modifié pour autoriser le droit d'usage à but lucratif. Cela ne changera pas la pratique actuelle, mais conjointement avec la propriété coutumière évoquée ci-dessus, cela formalisera au moins la vente et le bois finalement vendu sur le marché domestique. Cette mesure devra être associée à celles mentionnées plus haut sur la délivrance et le contrôle de permis de coupe adaptés.

Problème II manque au marché domestique une niche spécifique pour les autorisations PEBO.

\section{Recommandation}

- L'accès au PEBO (ou à son équivalent amélioré à l'avenir) devrait être relié à l'existence d'un accord de partenariat avec un négociant enregistré sur le marché domestique du bois. Ces accords existent déjà entre les négociants et les scieurs artisanaux, mais ils ne sont pas officiels; souvent verbaux et sujets à dispute. S'ils étaient rendus obligatoires pour demander un permis de coupe annuel, ils pourraient aider l'administration à suivre et contrôler les activités d'exploitation et de vente, sans augmenter les coûts de transaction pour les opérateurs.

Problème Les scieurs artisanaux ont été généralement exclus des négociations APV. Cela est dû en partie à leur éparpillement sur un vaste territoire et à une capacité d'organisation médiocre, ainsi qu'aux tensions qui existent souvent entre la volonté affichée des pouvoirs publics de les faire sortir du secteur informel et le comportement de fonctionnaires décentralisés à la recherche de rentes.

\section{Recommandation}

- $\quad$ Tirer parti des structures déjà en place (p. ex. les syndicats internes sur chaque marché, comme Pro-Formal l'a documenté), mais favoriser leur fédération en entités régionales ou nationales plus importantes.

Problème II n'y a pas de demande pour le bois légal sur le marché domestique ou régional.

\section{Recommandation}

- Le MINFOF devrait mettre la pression sur le gouvernement pour qu'il adopte une politique publique exigeant que tous les appels d'offres publics (incluant notamment ceux financés par l'aide extérieure ou les projets de développement pour l'infrastructure, la santé ou l'éducation) prévoient uniquement l'utilisation de bois légal. 


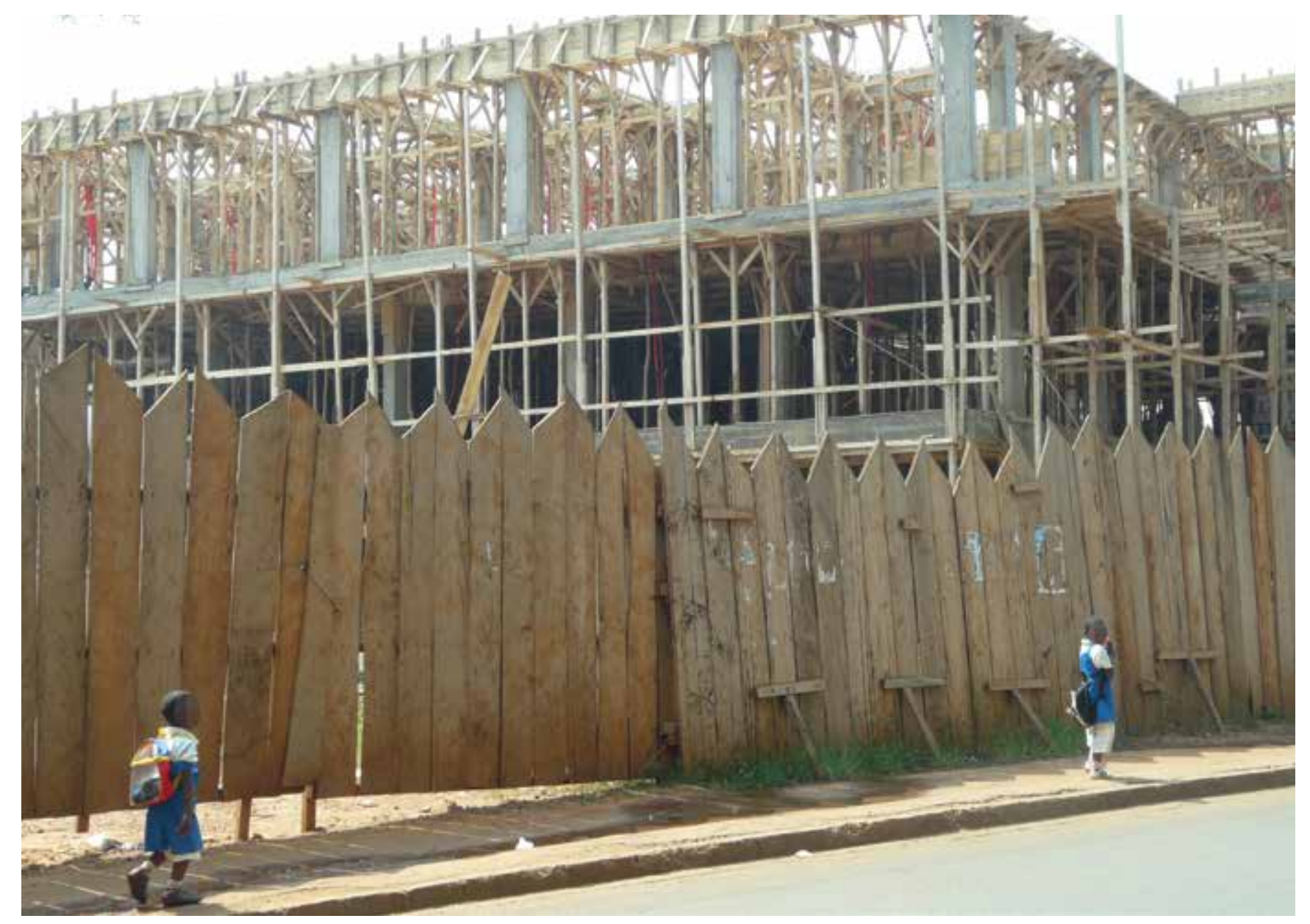

Bois utilisé comme matériel de construction, Cameroun

Photo Paolo Omar Cerutti, CIFOR

Problème Le secteur forestier industriel n'utilise qu'un petit pourcentage des coupes annuelles autorisées dans les concessions, mais les petits opérateurs ne peuvent pas accéder à ce bois, ce qui les force à exploiter pratiquement exclusivement les arbres situés dans les zones agroforestières.

\section{Recommandations}

- Amorcer des discussions officielles au sein du MINFOF sur le meilleur moyen de faire correspondre une offre potentielle de bois (officiellement exploité durablement dans les concessions) à une demande assurée, c.-à-d. le marché domestique du bois.

- $\quad$ Si les solutions techniques sont difficiles à mettre en œuvre (p. ex. pour autoriser les scieurs artisanaux à pénétrer dans les concessions forestières), on pourrait recourir à des incitations financières destinées aux sociétés industrielles qui se porteraient volontaires pour transporter ce bois hors des concessions, ou aux scieurs artisanaux qui mettraient en place un partenariat enregistré avec des industriels.

Problème L'absence d'information sur le prix du bois dans la plupart des zones rurales provoque des relations de pouvoir inégales entre acheteurs et vendeurs.

\section{Recommandation}

- Une palette d'outils de communication (p. ex. radios locales ou SMS) devrait être utilisée pour informer régulièrement les communautés rurales du prix du bois, par essence, sur les marchés urbains.

Problème II n'existe pas de politique fiscale pour le secteur domestique du bois.

\section{Recommandations}

- Adopter une politique fiscale spécifique pour le secteur, qui remédierait à tous les points névralgiques de la chaîne, de l'exploitation à la consommation. Cette politique devrait être (au moins à court terme) de nature incitative, pour éviter d'être perçue par les opérateurs comme un nouveau fardeau imposé par l'APV.

- D'autre part, des sanctions bien définies doivent être mises en place pour les fonctionnaires qui se livrent à des extorsions de fonds auprès des opérateurs. À cet effet, le MINFOF devrait publier un document détaillant clairement les sanctions pour chaque type d'inconduite de ses agents.

- L'introduction de sanctions pourrait être associée à des mesures incitant les fonctionnaires provinciaux à mettre un 
terme à leurs anciennes habitudes. Un exemple d'incitation pourrait être la mise en place de primes de performance qui seraient financées par des fonds collectés par la lutte contre la fraude.

\section{Bibliographie}

République du Cameroun et Union européenne. 2010 Voluntary partnership agreement between the European Union and the Republic of Cameroon on forest law enforcement, governance and trade in timber and derived products to the European Union (FLEGT). République du Cameroun et Union européenne, Yaoundé et Bruxelles.

Cerutti PO et Lescuyer G. 2011 The domestic market for smallscale chainsaw milling in Cameroon: Present situation, opportunities and challenges. Document occasionnel 61. Centre de recherche forestière internationale, Bogor, Indonésie. Version française aussi disponible : Cerutti PO et Lescuyer G. 2011 Le marché domestique du sciage artisanal au Cameroun : État des lieux, opportunités et défis. Document occasionnel 65 . Centre de recherche forestière internationale, Bogor, Indonésie.] Cerutti PO et Tacconi L. 2008 Forests, illegality, and livelihoods: The case of Cameroon. Society \& Natural Resources 21(9):845-53.
Cerutti PO, Tacconi L, Lescuyer G et Nasi R. 2013 Cameroon's hidden harvest: Commercial chainsaw logging, corruption and livelihoods. Society \& Natural Resources 26(5):539-53.

CIFOR et MINFOF. 2013 Etude de l'importance économique et sociale du sous-secteur forêt-faune au Cameroun. Centre de recherche forestière internationale (CIFOR), Bogor, Indonésie et Ministère des Forêts et de la Faune (MINFOF), Yaoundé.

\section{Lectures complémentaires}

Kishor N et Lescuyer G. 2012 Controlling illegal logging in domestic and international markets by harnessing multilevel governance opportunities. International Journal of the Commons 6(2):255-70.

Putzel L, Kelly A, Cerutti PO, Artati Y. 2014 Formalization of natural resource access and trade: Insights from land tenure, mining, fisheries, and non-timber forest products. Centre de recherche forestière internationale, Bogor, Indonésie.

Wit M, van Dam J, Cerutti PO, Lescuyer G, Kerrett R et Parker Mckeon J. 2011 Chainsaw milling: Supplier to local markets - A synthesis. Dans : Wit M et van Dam J, éds. Chainsaw milling: Supplier to local markets. Tropenbos International, Wageningen, Pays-Bas. VII-XXII.

Le projet Pro-Formal (EuropeAid/ENV/2010-242904/TPS), financé par I'UE, a été mis en œuvre par le Centre de recherche forestière internationale (CIFOR) dans trois régions (Asie du Sud-Est, Afrique subsaharienne et Amérique latine) et cinq pays (Indonésie, Cameroun, République démocratique du Congo, Gabon et Équateur) de juillet 2010 à décembre 2013. Le projet s'est déroulé avec plusieurs partenaires clés, et certains résultats ont déjà été diffusés par le CIFOR grâce à ses documents occasionnels, Infobriefs, articles publiés dans des revues scientifiques et autres articles sur internet (consultables sur le site internet du projet : www.cifor.org/pro-formal).

\section{(b) RESEARCH RESEAT ON Forests, Trees and Agroforestry}

Cette recherche a été menée par le CIFOR dans le cadre du Programme de recherche du CGIAR sur les forêts, les arbres et l'agroforesterie (CRP-FTA). Ce programme collaboratif vise à améliorer la gestion et l'utilisation des forêts, de l'agroforesterie et des ressources génétiques des arbres à l'échelle du paysage, des forêts aux exploitations agricoles. Le CIFOR dirige le CRP-FTA en partenariat avec Bioversity International, le CATIE, le CIRAD, le Centre international d'agriculture tropicale et le Centre mondial de l'Agroforestrie.

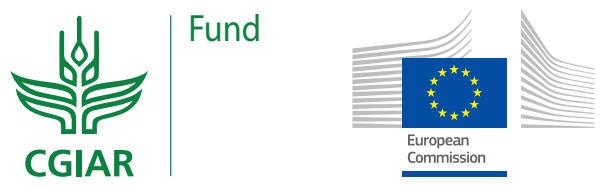

